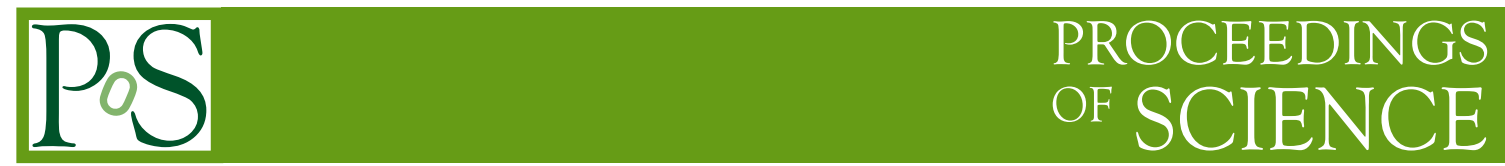

\title{
Measurement of Beauty and Charm Photoproduction at HERA
}

\author{
Karin Daum*t \\ Bergische Universität Wuppertal, Gaußstraße 20, D-42097 Wuppertal, Germany \\ E-mail: karin.daumedesy. de
}

\section{On behalf of the $\mathrm{H} 1$ collaboration}

Recent measurements of open beauty and charm photoproduction cross sections from the H1 Collaboration at HERA are summarised. Results from two new analyses are presented which are based on the identification of heavy quark production via semi-leptonic decays of heavy flavour hadrons. Leading and next-to-leading order QCD predictions are compared to these measurements.

XXI International Workshop on Deep-Inelastic Scattering and Related Subjects -DIS2013, 22-26 April 2013

Marseille, France

\footnotetext{
*Speaker.

${ }^{\dagger}$ Permanent address: DESY, Notkestarße 85, D-22603 Hamburg, Germany
} 


\section{Introduction}

The measurement of open beauty and charm production in $e p$ scattering at HERA is an important testing ground for perturbative Quantum Chromodynamics (pQCD). The masses $m_{b}$ and $m_{c}$ of the beauty and charm quarks provide a sufficiently hard scale for applying pQCD in cross section calculations. The dominant beauty and charm production process at HERA is direct boson-gluonfusion (BGF), $\gamma p \rightarrow Q \bar{Q} X$, with $Q=c, b$, in which a virtual photon emitted from the electron ${ }^{1}$ fused with a gluon emitted from the proton via the creation of a $q \bar{q}$ pair (figure 1a). In photoproduction, with photon virtuality $Q^{2} \approx 0$, the scattering process can be viewed as scattering off a quasi-real photon target. The photon can fluctuate into a hadronic structure from which one of the emitted partons takes part in the hard interaction (figure 1b). Processes of this kind are termed resolved photoproduction. A strict separation into direct and resolved processes is only possible in leading order (LO) QCD which predicts significant contributions from resolved photons to the photoproduction cross section.
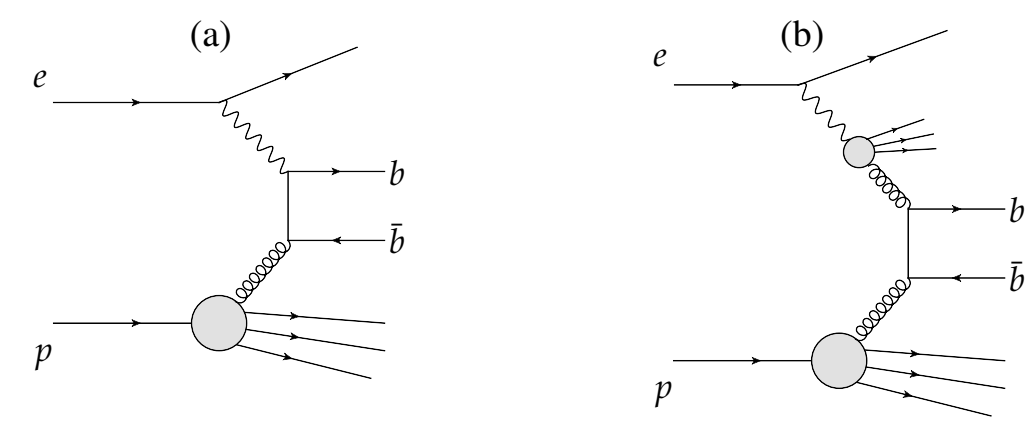

Figure 1: Generic leading order diagrams for heavy quark production in $e p$ collisions. The diagram (a) is referred to as direct, the diagram (b) is referred to as resolved.

New results from the H1 Collaboration on beauty and charm photoproduction at HERA from two different analyses $[1,2]$ are presented. Both analyses exploit semi-leptonic decays from heavy flavoured hadrons for tagging beauty and charm events. In the first analysis charm and beauty production is measured using identified muons in di-jet events [1]. Due to the requirements on the transverse momenta of the jets of $P_{T}^{\text {jet } 1}>7 \mathrm{GeV}$ and $P_{T}^{\text {jet } 2}>6 \mathrm{GeV}$ for the most energetic and the second jet, respectively, this analysis is sensitive to charm and beauty production significantly above the respective production thresholds. The second analysis [2] is specifically tailored to explore beauty production at threshold and is based on events with two electrons with transverse momentum $P_{T}(e)>1 \mathrm{GeV}$ using a novel technique based on low momentum electron identification [3]. This analysis became only possible because a dedicated trigger was developed which identifies low momentum electrons already online by combining 3D-tracks from the Fast Track Trigger [3] (FTT) with low energy deposition in the calorimeter reconstructed by the Jet Trigger [4] (JT).

\section{Charm and beauty photoproduction using semi-muonic decays in di-jet events}

The data were collected with the H1 detector [5] in the years 2006 and 2007 and correspond to an integrated luminosity of $\mathscr{L}=179 \mathrm{pb}^{-1}$. Electrons of $27.6 \mathrm{GeV}$ were colliding with protons of

\footnotetext{
${ }^{1}$ The term 'electron' will be used for electrons and positrons in not stated otherwise.
} 

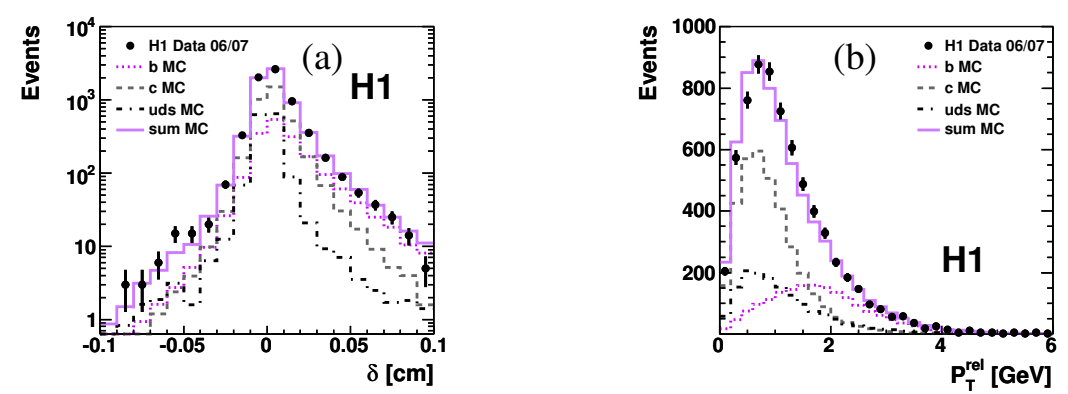

Figure 2: The impact parameter $\delta$ and $P_{T}^{\text {rel }}$ distributions for the total event sample. The data are compared to the PYTHIA predictions of the different quark contributions and their sum.

$920 \mathrm{GeV}$ resulting in an $e p$ centre-of-mass energy of $\sqrt{s} \approx 320 \mathrm{GeV}$. Photoproduction events are selected requiring the absence of an isolated electromagnetic cluster in the calorimeters consistent with a signal from a scattered electron. This limits the photon virtuality to values of $Q^{2}<2.5 \mathrm{GeV}^{2}$. The inelasticity $y$, reconstructed from the hadronic final state (HFS) is restricted to $0.2<y<0.8$.

Muon candidates are identified as track segments reconstructed in the instrumented iron which are matched with tracks reconstructed in the central tracking detector (CTD). At least two hits measured in the central silicon tracker have to be associated to the muon track. Events are selected with at least one muon candidate reconstructed in the instrumented iron having a pseudorapidity within $-1.3<\eta_{\mu}<1.5$ and a transverse momentum of $P_{T}^{\mu}>2.5 \mathrm{GeV}$.

Jets are reconstructed in the laboratory frame using the inclusive longitudinally invariant $k_{T}$ algorithm [6] in the massless $P_{T}$ recombination scheme. Events with at least two jets are selected, where the leading two jets are required to be in the angular range $-1.5<\eta^{\text {jet }}<2.5$ and to have a transverse momentum of $P_{T}^{\text {jet } 1}>7 \mathrm{GeV}$ and $P_{T}^{\text {jet } 2}>6 \mathrm{GeV}$. The muon has to be associated to one of the two most energetic jets.

The flavour of an event is defined as the hadron flavour of the $\mu$-jet. For the flavour separation two specific properties of heavy flavoured hadrons are exploited: their large masses and their longevity. These are reflected in the following properties of the muon track originating from semimuonic decays of heavy flavoured hadrons: (a) the signed impact parameter $\delta$ of the muon, which is the distance of closest approach of the muon track to the primary vertex, is expected to be positive and (b) the transverse momentum $P_{T}^{r e l}$ relative to the jet axis is expected to be large. The measured distributions of these two observables are shown in figure 2 together with the expectations from light flavours (uds), charm (c) and beauty (b) PYTHIA [7] Monte Carlo (MC) simulations. Differences in shape of the different MC templates are evident. The fraction of events with beauty, charm and light quarks, $f_{b}, f_{c}$ and $f_{l}$, are determined by binned likelihood fits of templates in the $\delta-P_{T}^{r e l}$ plane. The fitted fractions $f_{b}$ and $f_{c}$ are then converted to beauty and charm cross sections. This procedure is applied for the determination of the total visible and of the differential cross sections. The measured cross sections are proportional to the rate of events with a muon and a dijet system.

In figure 3 differential beauty and charm cross sections in the photoproduction regime are shown as a function of $P_{T}^{\mu}$ and $x_{\gamma}^{o b s}$ in comparison with LO and NLO predictions. The observable $x_{\gamma}^{o b s}$ is reconstructed from the particles assigned to the two leading jets and the HFS, i.e. $x_{\gamma}^{o b s}=\left(\sum_{\text {jet } 1}\left(E-P_{z}\right)+\sum_{j e t 2}\left(E-P_{z}\right)\right) / \sum_{H F S}\left(E-P_{z}\right)$, where $E$ and $P_{z}$ denote the energy and the 

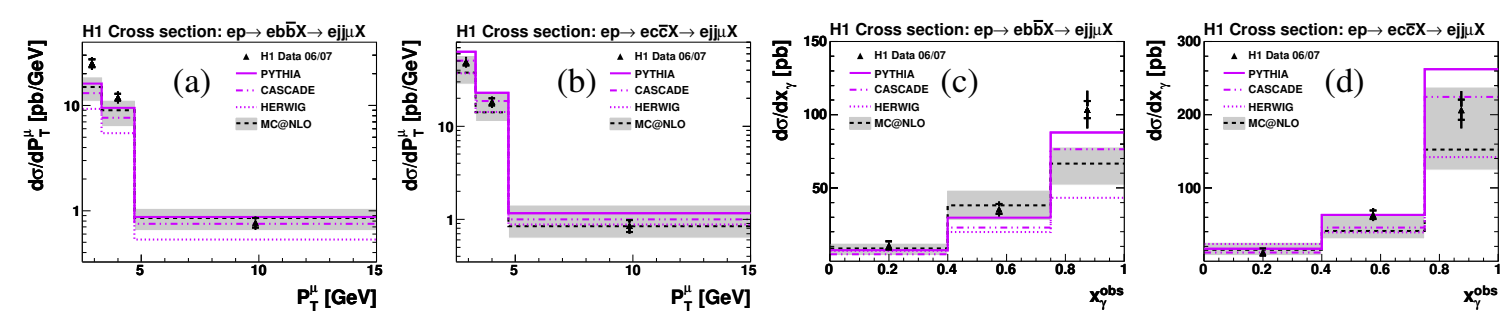

Figure 3: Measured differential $(\mathrm{a}, \mathrm{c})$ beauty and $(\mathrm{b}, \mathrm{d})$ charm production in the photoproduction regime as a function of $(\mathrm{a}, \mathrm{b})$ the transverse momentum $P_{T}^{\mu}$ and $(\mathrm{c}, \mathrm{d}) x_{\gamma}^{o b s}$. The data are compared to different LO and NLO predictions.

longitudinal projection of the momentum onto the proton beam direction of the particles. In the LO picture contributions to the cross section from direct and resolved photon processes are expected to have values of $x_{\gamma}^{o b s} \approx 1$ and $x_{\gamma}^{o b s} \ll 1$, respectively. The data show a larger relative contribution from resolved photon processes in beauty compared to charm production. The data are reasonably well described by the MC models and the MC@NLO [8] calculation with the exception of HERWIG [9] which is low in normalisation. For charm production the central prediction of MC@NLO fall also below the data. The experimental uncertainties of the measurements are in general significantly smaller than the theoretical uncertainties in the NLO calculations, especially in case of charm production.

\section{Beauty photoproduction near threshold using di-electron events}

The data sample used for this analysis was collected in the year 2007 when electrons with an energy of $27.6 \mathrm{GeV}$ collided with protons of $920 \mathrm{GeV}$, and when the FTT and JT were in operation on all trigger levels. The recorded data correspond to an integrated luminosity of $48.1 \mathrm{pb}^{-1}$. Similar selection criteria than for the analysis presented in section 2 are applied to select photoproduction events. The phase space is limited to the region $Q^{2}<2 \mathrm{GeV}^{2}$ and $0.05<y<0.65$.

This measurement is based on the identification of two low momentum electrons in the LAr calorimeter with $P_{T}^{e}>$ $1 \mathrm{GeV}$ to tag semi-electronic decays in beauty photoproduction. The electrons can either originate from the same $b$-quark $\left(b \rightarrow c e^{-} \bar{v}_{e} \rightarrow s e^{-} e^{+} \bar{v}_{e} v_{e}\right)$ or from different $b$ quarks. Therefore the electrons can either be of opposite or of same charge. Background to $b \bar{b}$ production arises form open charm or $J / \psi$ production, where in both cases the electrons are of opposite charge, and mis-identified electrons mainly originating from light flavour production.
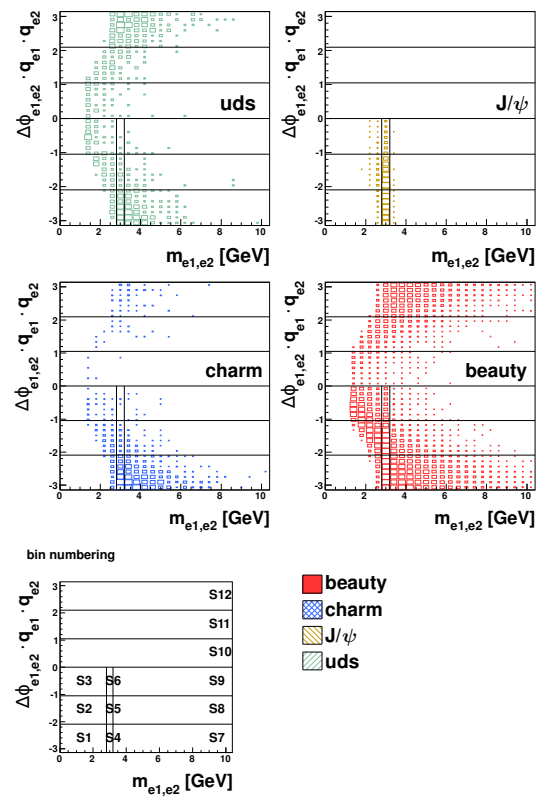

Figure 4: Templates in the $\Delta \phi_{e 1, e 2} \cdot q_{e 1}$. $q_{e 2}, m_{e 1, e 2}$ plane for $u d s, J / p s i, c$ and $b$ production obtained by MC simulation. 
The different contributions to this analysis can be disentangled by using the correlations in charge, invariant mass and opening angle of the electron pairs. In figure 4 the templates for $u d s, J / \psi$, charm and beauty production as obtained from MC simulation are shown for the $\Delta \phi_{e 1, e 2} \cdot q_{e 1} \cdot q_{e 2}-m_{e 1, e 2}$ plane. Electron pairs from $b \bar{b}$ production exhibit a signature different from the other samples: they have on average higher masses $m_{e 1, e 2}$, large opening angles $\phi_{e 1, e 2}$ and are almost equally shared between the 'same sign' and 'opposite sign' samples.

Using an regularised unfolding procedure the number of background events $N_{u d s}, N_{J / \psi}, N_{c}$ and the number of beauty events $N_{b}$ are derived in four bins of the average transverse momentum of the $b$-quark, $\left\langle P_{T}(b)\right\rangle$. The result is shown in figure 5 together with the NLO QCD expectation based on the FMNR program [10]. Good agreement is observed between the measurements and the NLO QCD calculation.

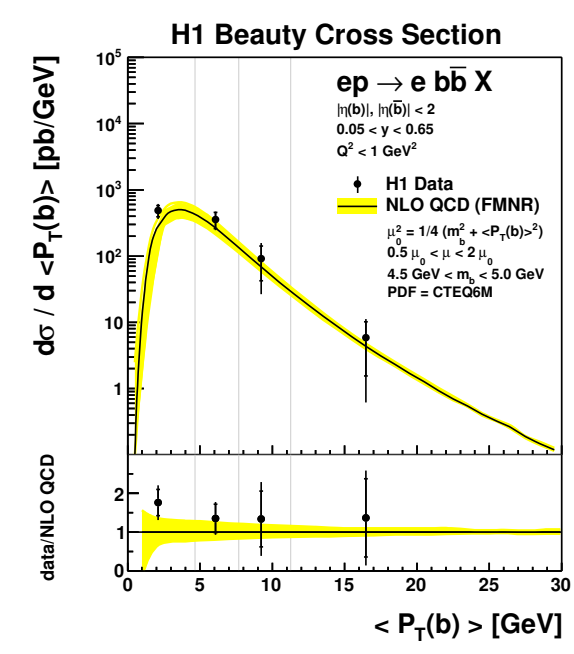

Figure 5: Differential beauty cross section $\mathrm{d} \sigma / \mathrm{d}\left\langle P_{T}(b)\right\rangle$ as a function of the quadratically added transverse momentum of the $b$-quarks $\left\langle P_{T}(b)\right\rangle$ in comparison to the FMNR NLO QCD calculation.

\section{Conclusions}

New results on beauty and charm photoproduction have been presented. Semi-leptonic decays of heavy flavoured hadrons are used for tagging beauty and charm production. Theory predictions agree reasonably well with the measurements within the still large theory uncertainties.

\section{References}

[1] F. D. Aaron et al.[H1Collaboration] Eur. Phys. J. C72 (2012) 2047 [arxiv:1205.2495].

[2] F. D. Aaron et al.[H1Collaboration] Eur. Phys. J. C72 (2012) 2148 [arxiv:1206.4346].

[3] M. Sauter, "Measurement of Beauty Photoproduction at Threshold using Di-Electron Events with the H1 Detector at HERA", Ph.D. thesis, ETH Zürich (2009), Diss. ETH No. 18652 and DESY-THESIS-2009-047 (available at http://www-h1.desy.de/publications/theses list.html).

[4] B. Olivier et al. Nucl. Instrum. Methods A641 (2011) 58.

[5] I. Abt et al.[H1 Collaboration] Nucl. Instrum. Methods A386 (1997) 310 and 348; R.D. Appuhn et al.[H1 SpaCal Group] Nucl. Instrum. Methods A386 (1997) 397.

[6] S.D. Ellis and D.E. Soper, Phys. Rev. D48 (1993) 3160 [hep-ph/9305266]; S. Catani et al. Nucl. Phys. B406 (1993) 187.

[7] T. Sjöstrand, S. Mrenna, and P.Z. Skands, JHEP 0605 (2006) 026 [hep-ph/0603175].

[8] T. Toll and S. Frixione, Phys. Lett. B703 (2011) 452 [arXiv:1106.1614].

[9] G. Corcella et al., JHEP 0101 (2001) 010 [hep-ph/0011363].

[10] S. Frixione et al., Adv. Ser. Direct. High Energy Phys. 15 (1998) 609 [hep-ph/9702287]. 